CERN-PH-TH/2008-246

RM3-TH/08-22

\title{
Flavor Changing Fermion-Graviton Vertices
}

\author{
G. Degrassi ${ }^{a, b}$, E. Gabrielli ${ }^{b}$, and L. Trentadue ${ }^{c}$ \\ a Dipartimento di Fisica, Università di Roma Tre and INFN, Sezione di Roma Tre, Via della \\ Vasca Navale 84, I-00146 Rome, Italy \\ b CERN PH-TH, CH-1211 Geneva 23, Switzerland \\ c Dipartimento di Fisica, Università di Parma and INFN, Gruppo Collegato di Parma, Viale \\ delle Scienze 7, I-43100 Parma, Italy
}

\begin{abstract}
We study the flavor-changing quark-graviton vertex that is induced at the one-loop level when gravitational interactions are coupled to the standard model. Because of the conservation of the energy-momentum tensor the corresponding form factors turn out to be finite and gauge-invariant. Analytical expressions of the form factors are provided at leading order in the external masses. We show that flavor-changing interactions in gravity are local if the graviton is strictly massless while if the graviton has a small mass long-range interactions inducing a flavor-changing contribution in the Newton potential appear. Flavor-changing processes with massive spin-2 particles are also briefly discussed. These results can be generalized to the case of the lepton-graviton coupling.
\end{abstract}




\section{Introduction}

Gravity is the only fundamental interaction which is universally coupled to all matter fields. Universality is then guaranteed by the tree-level coupling of the graviton field with the conserved energy-momentum tensor. However, when quantum corrections are taken into account, mattercoupling universality could be spoiled.

It is known that quantum corrections to the graviton-matter vertices (without gravitons in the loops) are finite if the underlying theory of matter fields (in flat space-time) is renormalizable [1, 2, 3, 4, 5, 6]. This remarkable property is just a consequence of the Ward Identities (WI) that result from the energy-momentum conservation [3]. Therefore, (matter) radiative corrections to the graviton-matter vertices can be consistently calculated in the framework of gravity coupled to the standard model (SM) theory.

Long ago, Berends and Gastmans evaluated the finite corrections to the graviton-photon and graviton-electron vertices due to virtual quantum electrodynamical (QED) processes at the one-loop leve* [6]. In the latter case the corrections found lead to a modification of the Newton's law. In particular, for an electron (or any charged particle) in a gravitational field of a stationary mass $M$, they found a repulsive correction term proportional to the classical radius of the particle, $r_{e}=\alpha / m_{e}$, or

$$
V(r) \sim-\frac{G_{N} M m_{e}}{r}\left(1-\frac{\alpha}{2 m_{e} r}\right)
$$

where $G_{N}$ is the Newton constant, $\alpha$ is the fine-structure constant, and $m_{e}$ is the mass of the electron. At macroscopic distances $\left(r \gg r_{e}\right)$ the modification of Newton's law induced by the $1 / r^{2}$ term in Eq.(11) is extremely small, $\mathcal{O}\left(10^{-22}\right)$ at the surface of the earth [6].

The term $1 / r^{2}$ has an infrared origin as it can be simply understood by looking at the singular behavior of the one-loop Feynman diagrams in the massless limit $\left(m_{e} \rightarrow 0\right)$. The correction to the tree-level electron-graviton vertex is proportional to $\alpha \sqrt{-q^{2} / m_{e}^{2}}$ for negative small values of $q^{2}$, where $q$ is the momentum transfer. This is due to the fact that the photon has a tree-level coupling with the graviton. Then, the diagram where the external graviton is attached to two virtual photons in the loop contains a term with a powerlike infrared singularity as $m_{e} \rightarrow 0$. Thus, after Fourier transforming into coordinates space, this contribution gives rise to a $1 /\left(r^{2} m_{e}\right)$ correction to the Newton potential. Notice that there is no counterpart of such effect in QED. Indeed, due to the absence of self-photon interactions at tree-level, the Coulomb potential is protected against $1 / r^{2}$ corrections of order $\mathcal{O}(\alpha)$.

*The same calculation was previously done by Delbourgo and Phocas-Cosmetatos in [1], but it was incorrect due to the wrong Feynman rules adopted [6]. 
It is remarkable that, although derived in the framework of quantum field theory, the $1 / r^{2}$ term in Eq.(1) has a pure classical origin and it was first obtained on the basis of purely classical considerations in Ref.[7]. Indeed this term arises from the fact that the total mass of the particle is not concentrated at a point but is partly distributed as field energy in the space around the particle [7].

When gravity is coupled to the weak interactions the one-loop radiative corrections to the graviton-fermion vertex will include also the virtual exchanges of the weak gauge bosons $W^{ \pm}$ and $Z$. At the moment, a complete study of these effects is still missing. These corrections are expected to give small contributions in the low energy limit and furthermore to be infrared safe, being the $W^{ \pm}$and $Z$ massive. Therefore, no long-distance modifications in the Newton potential are expected from the weak radiative corrections.

A peculiar aspect of the weak interactions is that they can induce (at one loop) flavorchanging neutral currents (FCNC) processes in the fermionic sector. Since FCNC are absent at tree-level, they arise as a pure quantum effect. Analogously, flavor-changing (FC) graviton vertices are not present at the tree-level. However, when gravity is coupled to the weak interactions, quantum weak-corrections can induce an off-diagonal contribution (in flavor space) to the energy-momentum tensor. These effects are the spin-2 counterpart of the standard spin-1 FCNC contribution in the SM. Because of the tensorial nature of the coupling of spin-2 particles to the matter fields we will refer to these effects as tensorial flavor-changing neutral currents (TFCNC).

The aim of this paper is to compute the form factors of the TFCNC and analyze their gravitational couplings. We perform the exact computation of the one-loop FC fermion-graviton vertex, i.e. retaining the full dependence on all masses and momenta. We investigate under which conditions these effective couplings could induce modifications in the Newton potential discussing both cases of massless and massive-graviton exchange. We consider a few applications of these results in the case of massive spin-2 particles. In particular, we analyze the FC decay $f_{1} \rightarrow f_{2} G$, and the decay $G \rightarrow f_{1} \bar{f}_{2}$ where $G$ stands for a massive spin-2 particle. Our results can be easily generalized to the leptonic sector with massive Dirac neutrinos, provided the CKM matrix of the quark sector is substituted with the corresponding leptonic one.

The paper is organized as follows. In section 2 we specify the interaction between the SM fields and the graviton by suitably choosing a gauge that avoids the appearance of cubic vector boson-Goldstone-graviton interactions. We analyze the general structure of the offdiagonal matrix element (in flavor space) of the energy-momentum tensor of fermion fields, derive the corresponding Ward-identities, and provide the analytical expressions of the relevant form factors. The analysis of the gravitational couplings of the TFCNC is carried out in Sec. 3. In Sec. 4 we analyze a few applications of these results. Finally, Sec. 5 contains our conclusions. 


\section{Flavor-changing quark-graviton vertex}

We are going to evaluate the interaction between the graviton and two generic fermion states with different flavor or the off-diagonal matrix element of the energy-momentum tensor $T^{\mu \nu}(x)$. In particular, working in the basis of mass-eigenstates for the fermion fields, we are interested in calculating the following matrix element

$$
\hat{T}^{\mu \nu} \equiv-i\left\langle p_{2}\left|T^{\mu \nu}(0)\right| p_{1}\right\rangle,
$$

where the initial and final states are assumed to have momenta $p_{1}$ and $p_{2}$ respectively with $p_{i}^{2}=m_{i}^{2}$ and spinorial wave-functions $u_{i}\left(p_{i}\right)(i=1,2)$ while the four-momenta $p, q$ are defined as $p=p_{1}+p_{2}$ and $q=p_{1}-p_{2}$. It is understood that initial and final states have different flavor and mass. The relevant Feynman rules which are necessary in order to calculate the above matrix element, can be easily derived by looking at the graviton couplings with matter fields.

The interactions between gravity and the SM fields are assumed to be described by the action integral

$$
\mathcal{S}=\int d^{4} x \sqrt{-\mathrm{g}} \mathcal{L}_{\mathrm{SM}}\left[g_{\mu \nu}\right]
$$

where the SM Lagrangian, $\mathcal{L}_{\mathrm{SM}}$, is thought to be written in terms of the metric tensor $g_{\mu \nu}$ and for the fermionic part using the vierbein formalism. In Eq.(3) $\mathrm{g}=\operatorname{det} g_{\mu \nu}$ and $\mathcal{L}_{\mathrm{SM}}$ includes the classical term and the gauge-fixing function in the $R_{\xi}$ gauge (the term with the ghost fields is not relevant for our discussion). To obtain the interaction of the graviton with the SM fields we expand in Eq.(3) the metric $g_{\mu \nu}$ around the flat one as $g_{\mu \nu}=\eta_{\mu \nu}+\kappa h_{\mu \nu}$, retaining only the first term in the graviton field where $\eta_{\mu \nu}=(+1,-1,-1,-1), \kappa=\sqrt{32 \pi G_{N}}$, and $h_{\mu \nu}$ is interpreted as the spin-2 graviton field. We notice that, if in the gauge-fixing term in $\mathcal{L}_{\mathrm{SM}}$ the standard 't-Hooft gauge-fixing function is taken, vertices in which a graviton field induces a transition between a vector boson and its unphysical Goldstone boson counterpart are going to appear [8]. In order to avoid the appearance of vector-Goldstone-graviton interactions we find it convenient to use a modified version of the 't-Hooft gauge-fixing function, or

$$
\begin{aligned}
\mathcal{L}_{g . f .}= & -\frac{1}{2 \xi}\left[g_{\mu \nu} \partial^{\mu} W_{a}^{\nu}+g^{\nu \rho} \Gamma_{\nu \rho}^{\mu} W_{a \mu}+i \frac{g \xi}{2}\left(\phi^{\prime \dagger} \sigma^{a}\langle\phi\rangle-\left\langle\phi^{\dagger}\right\rangle \sigma^{a} \phi^{\prime}\right)\right]^{2} \\
& -\frac{1}{2 \xi}\left[g_{\mu \nu} \partial^{\mu} B^{\nu}+g^{\nu \rho} \Gamma_{\nu \rho}^{\mu} B_{\mu}+i \frac{g^{\prime} \xi}{2}\left(\phi^{\prime \dagger}\langle\phi\rangle-\left\langle\phi^{\dagger}\right\rangle \phi^{\prime}\right)\right]^{2}
\end{aligned}
$$

that differs from the standard one by the terms proportional to $\Gamma_{\nu \rho}^{\mu}$. In Eq.(44) $\xi$ is the gauge parameter, $g, W_{a}^{\mu}\left(g^{\prime}, B^{\mu}\right)$ are the coupling constant and fields of the $S U(2)(U(1))$ group, $\sigma^{a}$

$\dagger$ The definition of $\hat{T}^{\mu \nu}$ corresponds to the Feynman rule for the off-diagonal matrix element of the energymomentum tensor.

$\ddagger$ The addition of a term proportional to the Christoffel symbol in the gauge-fixing function of a spin-1 
are the Pauli matrices,

$$
\Gamma_{\nu \rho}^{\mu}=\frac{1}{2} g^{\mu \alpha}\left(\partial_{\rho} g_{\alpha \nu}+\partial_{\nu} g_{\alpha \rho}-\partial_{\alpha} g_{\nu \rho}\right)
$$

is the Christoffel symbol, and

$$
\phi^{\prime}=\left(\begin{array}{c}
\phi_{+} \\
\frac{\phi_{1}+i \phi_{2}}{\sqrt{2}}
\end{array}\right) \quad\langle\phi\rangle=\left(\begin{array}{c}
0 \\
\frac{v}{\sqrt{2}}
\end{array}\right)
$$

with $\phi_{1}$ the physical Higgs field, $\phi_{+}$and $\phi_{2}$ the unphysical counterparts of the $W^{+}$and $Z$ vector boson and $v$ the vacuum expectation value.

At the first order in the $\kappa$ expansion, the interaction Lagrangian for the tree-level graviton coupling to SM fields, is

$$
\mathcal{L}_{\text {int }}=-\frac{1}{\bar{M}_{P}} T^{\mu \nu} h_{\mu \nu}
$$

where $T^{\mu \nu}$ is the energy-momentum tensor of matter fields obtained from the Lagrangian in Eq.(3) with the gauge-fixing function given in Eq.(4). The mass-scale $\bar{M}_{P}$ appearing in Eq.(17) is the reduced Plank mass defined as $\bar{M}_{P} \equiv 2 / \kappa$. From Eq.(17), the Feynman rules can be derived. The ones, in the $R_{\xi}$ gauge, relevant for our calculation are collected in Appendix A. Feynman rules for graviton interactions with vector bosons, in the unitary gauge, can be found in Refs. [9, 10, 11].

Because of the $\mathrm{V}$-A nature of the charged weak currents, the exact expression of the offdiagonal matrix element (in flavor space) of energy-momentum tensor $\hat{T}^{\mu \nu}$ has the following structure

$$
\hat{T}^{\mu \nu}=\frac{i G_{F}}{16 \pi^{2} \sqrt{2}} \sum_{i=1}^{12} f_{i}(p, q) \bar{u}_{2}\left(p_{2}\right) O_{i}^{\mu \nu} u_{1}\left(p_{1}\right)
$$

with

$$
\begin{aligned}
& O_{1}^{\mu \nu}=\left(\gamma^{\mu} p^{\nu}+\gamma^{\nu} p^{\mu}\right) P_{L} \\
& O_{2}^{\mu \nu}=\left(\gamma^{\mu} q^{\nu}+\gamma^{\nu} q^{\mu}\right) P_{L} \\
& O_{3}^{\mu \nu}=\eta^{\mu \nu} M_{+} \\
& O_{4}^{\mu \nu}=p^{\mu} p^{\nu} M_{+} \\
& O_{5}^{\mu \nu}=q^{\mu} q^{\nu} M_{+} \\
& O_{6}^{\mu \nu}=\left(p^{\mu} q^{\nu}+q^{\mu} p^{\nu}\right) M_{+} \\
& O_{7}^{\mu \nu}=\eta^{\mu \nu} M_{-}
\end{aligned}
$$

massive particle was considered in Ref. [9]. However in that work the mechanism of mass-generation for the spin-1 particle was not addressed. 


$$
\begin{aligned}
O_{8}^{\mu \nu} & =p^{\mu} p^{\nu} M_{-} \\
O_{9}^{\mu \nu} & =q^{\mu} q^{\nu} M_{-} \\
O_{10}^{\mu \nu} & =\left(p^{\mu} q^{\nu}+q^{\mu} p^{\nu}\right) M_{-} \\
O_{11}^{\mu \nu} & =\frac{m_{1} m_{2}}{m_{W}^{2}}\left(\gamma^{\mu} p^{\nu}+\gamma^{\nu} p^{\mu}\right) P_{R} \\
O_{12}^{\mu \nu} & =\frac{m_{1} m_{2}}{m_{W}^{2}}\left(\gamma^{\mu} q^{\nu}+\gamma^{\nu} q^{\mu}\right) P_{R}
\end{aligned}
$$

where $P_{L, R}=\left(1 \mp \gamma_{5}\right) / 2$ and $M_{ \pm} \equiv m_{1} P_{R} \pm m_{2} P_{L}$.

The form factors appearing in Eq.(8) are not all independent. Indeed, due to the conservation of the energy-momentum tensor $\partial_{\mu} T^{\mu \nu}(x)=0$ (or equivalently $\partial_{\nu} T^{\mu \nu}(x)=0$ ), a subset of independent form factors arise. In momentum space, this relation is translated into

$$
q_{\mu}\left\langle p_{2}\left|T^{\mu \nu}(0)\right| p_{1}\right\rangle=0,
$$

which reduce the number of form factors $f_{i}(p, q)$ in Eq.(8) into an independent subset. Applying Eq.(10) into the right-hand side (r.h.s) of Eq.(88), we get the following set of Ward identities (WI)

$$
\begin{aligned}
(p \cdot q) f_{1}(p, q)+q^{2} f_{2}(p, q) & =0, \\
f_{3}(p, q)+q^{2} f_{5}(p, q)+(p \cdot q) f_{6}(p, q)+\frac{(p \cdot q)}{2 m_{W}^{2}} f_{12}(p, q) & =0, \\
(p \cdot q) f_{4}(p, q)+q^{2} f_{6}(p, q)+\frac{(p \cdot q)}{2 m_{W}^{2}} f_{11}(p, q) & =0, \\
f_{2}(p, q)+f_{7}(p, q)+q^{2} f_{9}(p, q)+(p \cdot q) f_{10}(p, q)-\frac{p^{2}+q^{2}}{4 m_{W}^{2}} f_{12}(p, q) & =0, \\
f_{1}(p, q)+(p \cdot q) f_{8}(p, q)+q^{2} f_{10}(p, q)-\frac{p^{2}+q^{2}}{4 m_{W}^{2}} f_{11}(p, q) & =0, \\
(p \cdot q) f_{11}(p, q)+q^{2} f_{12}(p, q) & =0 .
\end{aligned}
$$

These relations provide a strong check of our calculation in terms of Feynman diagrams.

In the case of light external states, the dominant contribution to $\hat{T}^{\mu \nu}$ comes from the $O_{1}$ and $O_{2}$ structures. Indeed the $O_{3}-O_{10}$ structures have a single chiral suppression while $O_{11}$ and $O_{12}$ are double chirally suppressed.

The interaction of the graviton with two different fermion fields is described at the lowest order by the one-loop diagrams drawn 8 in Fig.1. To be definite we assume the external fermion states to be quarks of different generation, then the mixing matrix entering in the $W^{+}$and $\phi^{+}$ vertices is the CKM matrix. We evaluated exactly, namely keeping the complete dependence

\footnotetext{
${ }^{\S}$ Feynman diagrams have been drawn using JaxoDraw [12]
} 

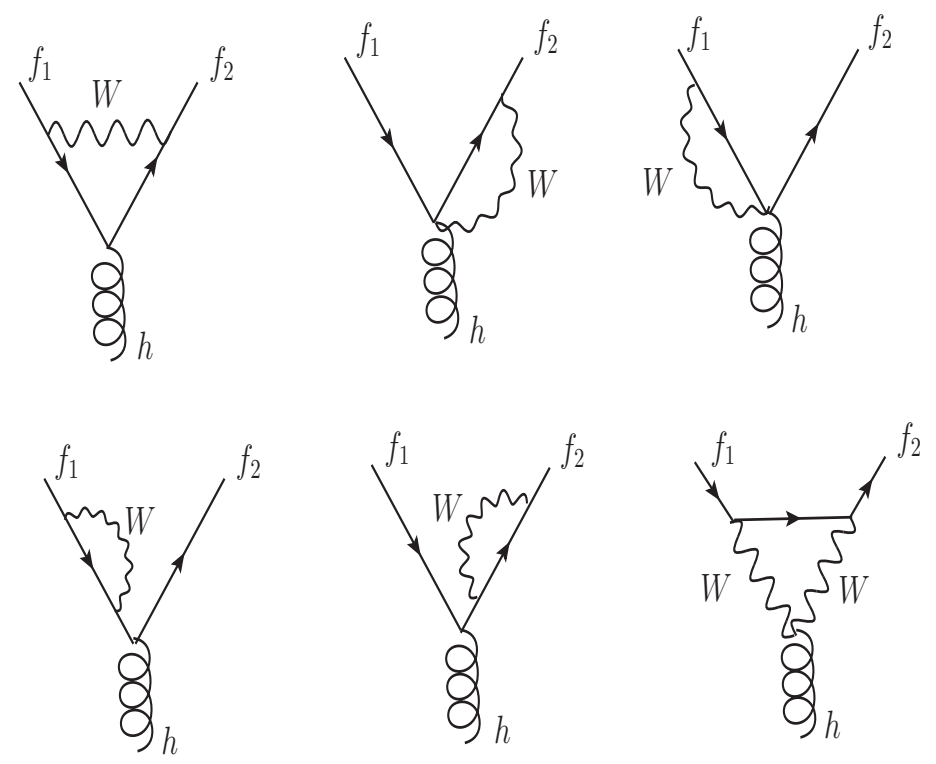

Figure 1: The one-loop Feynman diagrams contributing to the flavor-changing quark-graviton vertex in the unitary gauge. In the $R_{\xi}$ gauge there is also the contribution of diagrams where the $W$ boson is replaced by its unphysical counterpart.

on the $p, q$ momenta and on the internal/external masses, the Feynman diagrams in Fig.1 to obtain the form factors $f_{i}(p, q)$ appearing in Eq.(8). The calculation has been performed in the 't Hooft-Feynman gauge $(\xi=1)$ and cross-checked in the unitary gauge. While in the 't Hooft-Feynman gauge the result is finite before the application of the GIM mechanism, in the unitary gauge the GIM is needed to cancel some residual divergent term. From our result we explicitly verified all the WI reported in Eq.(11). It should be pointed out that to satisfy the WI the $\xi$-dependent term in the $W^{+} W^{-}$graviton vertex (see Eq.(42) in Appendix A) was necessary.

The complete result is too long to be reported, however; since we are mainly interested in analyzing the effects of the TFCNC in low energy processes, we report here the leading term of the form factors $f_{i}(p, q)$ in a $q^{2} / m_{W}^{2}$ and $(p \cdot q) / m_{W}^{2}$ expansion of the matrix element, keeping the dependence on the internal fermion masses exact. In this approximation, the nonvanishing factors are given by

$$
\begin{aligned}
f_{1}(p, q) & =-q^{2} \sum_{f} \lambda_{f} g_{a}\left(x_{f}\right) \\
f_{2}(p, q) & =(p \cdot q) \sum_{f} \lambda_{f} g_{a}\left(x_{f}\right)
\end{aligned}
$$




$$
\begin{aligned}
f_{3}(p, q) & =q^{2} \sum_{f} \lambda_{f} g_{b}\left(x_{f}\right) \\
f_{5}(p, q) & =-\sum_{f} \lambda_{f} g_{b}\left(x_{f}\right) \\
f_{7}(p, q) & =-2(p \cdot q) \sum_{f} \lambda_{f} g_{a}\left(x_{f}\right) \\
f_{10}(p, q) & =\sum_{f} \lambda_{f} g_{a}\left(x_{f}\right) \\
f_{11}(p, q) & =-q^{2} \sum_{f} \lambda_{f} g_{m}\left(x_{f}\right) \\
f_{12}(p, q) & =(p \cdot q) \sum_{f} \lambda_{f} g_{m}\left(x_{f}\right)
\end{aligned}
$$

where $x_{f}=m_{f}^{2} / m_{W}^{2}$ with $m_{f}$ the mass of the fermion running into the loop and $\lambda_{f} \equiv K_{f 1} K_{f 2}^{\star}$ (the external quarks are assumed to be of the down type), with $K_{i j}$ the corresponding CKM matrix element. The functions $g_{i}(x)$ appearing in Eq.(12) are given by

$$
\begin{aligned}
g_{a}(x)=\frac{1}{36(x-1)^{4}}[ & 44-194 x+243 x^{2}-98 x^{3}+5 x^{4} \\
& \left.+6 x\left(2-15 x+10 x^{2}\right) \log (x)\right] \\
g_{b}(x)=\frac{1}{6(x-1)^{4}}[ & \left.8-14 x+21 x^{2}-14 x^{3}-x^{4}+2 x\left(4+3 x+2 x^{3}\right) \log (x)\right] \\
g_{m}(x)=\frac{1}{72(x-1)^{6}}[ & 6-83 x+200 x^{2}+12 x^{3}-142 x^{4}+7 x^{5} \\
& \left.-12 x\left(1+4 x-18 x^{2}-2 x^{3}\right) \log (x)\right]
\end{aligned}
$$

It is easy to check that, in this approximation, the form factors in Eq.(12) satisfy the WI in Eq.(11). From Eqs.(12), (13) one sees that the leading contribution to $\hat{T}^{\mu \nu}$ is given in terms of two functions, namely $g_{a}(x)$ and $g_{b}(x)$. Indeed the explicit expression of the leading terms in $\hat{T}^{\mu \nu}$ is

$$
\begin{aligned}
\hat{T}^{\mu \nu}=\frac{i G_{F}}{16 \pi^{2} \sqrt{2}} \sum_{f} \lambda_{f} \bar{u}\left(p_{2}\right)\{ & \left(\gamma^{\mu} p^{\nu}+\gamma^{\nu} p^{\mu}\right) P_{L}\left(-q^{2}\right) g_{a}\left(x_{f}\right)+\left(\gamma^{\mu} q^{\nu}+\gamma^{\nu} q^{\mu}\right) P_{L}(p \cdot q) g_{a}\left(x_{f}\right) \\
& -q^{\mu} q^{\nu} M_{+} g_{b}\left(x_{f}\right)+\eta^{\mu \nu}\left[-2(p \cdot q) M_{-} g_{a}\left(x_{f}\right)+q^{2} M_{+} g_{b}\left(x_{f}\right)\right] \\
& \left.+\left(p^{\mu} q^{\nu}+p^{\nu} q^{\mu}\right) M_{-} g_{a}\left(x_{f}\right)\right\} u\left(p_{1}\right)
\end{aligned}
$$

and gauge invariance, $q_{\mu} T^{\mu \nu}=0$, is easily verified using $q_{\mu} \bar{u}\left(p_{2}\right) \gamma^{\mu} P_{L} u\left(p_{1}\right)=\bar{u}\left(p_{2}\right) M_{-} u\left(p_{1}\right)$.

In Appendix B we present $\hat{T}^{\mu \nu}$ in the approximation $(p \cdot q)=p^{2}+q^{2}=0$ keeping the full dependence on $q^{2}$ and the internal masses. 


\section{Flavor-changing gravitational couplings}

In this section we analyze the matrix element of the TFCNC coupled to an external gravitational source at the tree-level. We consider the following gravitational scattering

$$
f_{1}\left(p_{1}\right)+T^{\mathrm{ext}} \rightarrow f_{2}\left(p_{2}\right)+T^{\mathrm{ext}}
$$

where $T_{\mu \nu}^{\text {ext }}$ indicates the energy-momentum tensor of the external source, assumed to be conserved $\left(\partial^{\mu} T_{\mu \nu}^{\text {ext }}=0\right)$, and $f_{1,2}\left(p_{1,2}\right)$ are two fermions of different flavor and masses, with associated four-momenta $p_{1,2}$ respectively. In momentum space, the corresponding one-graviton exchange amplitude is given by

$$
\mathcal{M}=\frac{1}{\bar{M}_{P}^{2}} \hat{T}_{\mu \nu} P_{h}^{\mu \nu \alpha \beta}\left(q^{2}\right) \hat{T}_{\alpha \beta}^{\mathrm{ext}}
$$

where $P_{h}^{\mu \nu \alpha \beta}\left(q^{2}\right)$ is the graviton propagator in momentum space, with $q=p_{1}-p_{2}$, and $\hat{T}_{\mu \nu}^{\text {ext }}$ is the Fourier transform of $T_{\mu \nu}^{\text {ext }}(x)$. It is understood that all indices are contracted with the Minkowski metric $\eta_{\mu \nu}$. In the Einstein theory, the graviton propagator, in a covariant gauge, is given by

$$
P_{h}^{\mu \nu \alpha \beta}\left(q^{2}\right)=\frac{i}{q^{2}-i \varepsilon} \frac{1}{2}\left(\eta^{\mu \alpha} \eta^{\nu \beta}+\eta^{\nu \alpha} \eta^{\mu \beta}-\eta^{\mu \nu} \eta^{\alpha \beta}+Q^{\mu \nu \alpha \beta}(q)\right)
$$

where the last term $Q^{\mu \nu \alpha \beta}(q)$, which is gauge dependent, is a tensor made by linear combinations of an even number of $q$ momenta with open indices, such as for instance $q^{\mu} q^{\nu} \eta^{\alpha \beta}$ or $q^{\mu} q^{\nu} q^{\alpha} q^{\beta}$. It is also possible to set $Q^{\mu \nu \alpha \beta}=0$ by a particular gauge choice [13]. Nevertheless, due to the conservation of the energy-momentum tensor, the contribution of $Q^{\mu \nu \alpha \beta}(q)$ vanishes when

contracted with $\hat{T}_{\mu \nu}$ or $\hat{T}_{\alpha \beta}^{\text {ext }}$, leading to a gauge-invariant result. By using the energy-momentum conservation, we can write

$$
\mathcal{M}=\frac{i}{\left(q^{2}-i \varepsilon\right) 2 \bar{M}_{P}^{2}}\left[2 \hat{T}^{\mu \nu} \hat{T}_{\mu \nu}^{\mathrm{ext}}-C \hat{T}_{\mu}^{\mu} \hat{T}_{\nu}^{\mathrm{ext} \nu}\right] .
$$

where we introduce, for later reference, the numerical factor $C$ that in the present case (massless graviton) is equal to one. Because of the fact that the r.h.s. of Eq.(18) is not vanishing, the scattering of a fermion on an external gravitational field can induce a FC transition.

Despite the presence of the $1 / q^{2}$ pole in Eq.(18), the FC gravitational transition turns out to be local. Indeed by using the leading contributions from Eq.(14) in Eq.(18) we find

$$
\begin{array}{r}
\mathcal{M}=\frac{G_{F}}{16 \pi^{2} \sqrt{2} \bar{M}_{P}^{2}} \sum_{f} \lambda_{f} \bar{u}\left(p_{2}\right)\left\{\left(\gamma^{\mu} p^{\nu}+\gamma^{\nu} p^{\mu}\right) P_{L} g_{a}\left(x_{f}\right)-\eta^{\mu \nu} M_{+}\left(C g_{a}\left(x_{f}\right)-\left(\frac{3}{2} C-1\right) g_{b}\left(x_{f}\right)\right)\right. \\
\left.+\eta^{\mu \nu} M_{-} \frac{(p \cdot q)}{\left(q^{2}-i \varepsilon\right)} g_{a}\left(x_{f}\right)[2+C(-2)]\right\} u\left(p_{1}\right) \hat{T}_{\mu \nu}^{\mathrm{ext}} .
\end{array}
$$


The second line in Eq.(19) is zero if $\mathrm{C}=1$ (massless graviton) showing that in the Einstein theory of general relativity the $1 / q^{2}$ pole cancels out. This is a general result that holds also in the exact case, as can be easily proved using the WI in Eq.(11).

As we can see from Eq.(19), gravity and weak interactions induce at one loop an effective local interaction for the $\Delta F=1$ flavor transitions. The leading contribution to the corresponding effective Hamiltonian is given by local operators of dimension eight. The characteristic scale of this effective theory is $\Lambda=\sqrt{M_{P} m_{W}} \sim 10^{10} \mathrm{GeV}$, which is 6 orders of magnitude below the grand unified theory (GUT) scale. However, with respect to the known dimension six operators in the $\Delta F=1$ effective Hamiltonian of weak interaction the TFCNC gravitational operators induce negligible effects because the scale $\Lambda$ is much higher and moreover it enters in Eq.(19) at the fourth power.

The fact that the $1 / q^{2}$ pole cancels out in the amplitude $\mathcal{M}$ in Eq.(18) for a massless graviton, can be simply understood in terms of angular-momentum conservation. Let us suppose that the numerator of $\mathcal{M}$ does not vanish in the $q^{2} \rightarrow 0$ limit. If this would be the case, then TFCNC could be directly coupled to a on-shell massless graviton $\left(q^{2}=0\right)$. In particular, a nonvanishing matrix element for the fermion decay $f_{1} \rightarrow f_{2} G$ would be possible, where $G$ is an on-shell graviton and $m_{f_{1}}>m_{f_{2}}$. However, the decay $f_{1} \rightarrow f_{2} G$ is forbidden by angularmomentum conservation as can be easily understood looking, in the rest frame of the decaying particle, at the conservation of the angular momentum along the momentum direction of the two final states. In this frame one can see that angular momentum conservation is unbalanced along this direction, since a massless graviton carries only helicity states \pm 2 , while fermions can only have helicity states $\pm 1 / 2$. Therefore, in the case of massless graviton, only contact terms in the amplitude $\mathcal{M}$ are allowed. Instead the fermion decay $f_{1} \rightarrow f_{2} G$ does not vanish if the graviton has a small mass. Indeed, the spin content of a massive graviton contains five independent polarization states, including, among the spin- 2 and spin- 1 polarizations, also a spin-0 one. Then, the on-shell transition $f_{1} \rightarrow f_{2} G$ is allowed by angular-momentum conservation due to the presence of the spin-0 graviton polarization.

We consider now a scenario in which the graviton has a very small mass $m_{G}$. In this case, the corresponding graviton propagator in the unitary gauge is given by [13]

$$
P_{G}^{\mu \nu \alpha \beta}\left(q^{2}\right)=\frac{i}{q^{2}-m_{G}^{2}-i \varepsilon} \frac{1}{2}\left(\tilde{\eta}^{\mu \alpha} \tilde{\eta}^{\nu \beta}+\tilde{\eta}^{\nu \alpha} \tilde{\eta}^{\mu \beta}-\frac{2}{3} \tilde{\eta}^{\mu \nu} \tilde{\eta}^{\alpha \beta}\right)
$$

where $\tilde{\eta}^{\mu \nu} \equiv \eta^{\mu \nu}-q^{\mu} q^{\nu} / m_{G}^{2}$. The terms proportional to $q / m_{G}$, that in principle can be very enhanced, actually vanish when contracted with $T_{\mu \nu}$ and therefore do not contribute to the analogous transition amplitude $\mathcal{M}$ in Eq.(18). We assume here that $m_{G}$ is small enough that the corresponding Newton potential $\left(V(r) \sim \frac{1}{r} e^{-r m_{G}}\right)$ is not affected by its effect. This is clearly true for distances $r \ll 1 / m_{G}$, where $V(r) \sim 1 / r$. 
We recall that, apart from terms proportional to the momentum $q$, the massive-graviton propagator in Eq.(20) differs from the one of the Einstein theory in Eq.(17) by a finite term proportional to $\eta^{\mu \nu} \eta^{\alpha \beta}$ which does not vanish in the limit $m_{G} \rightarrow 0$. This is a consequence of the fact that the spin-0 component of the graviton field does not decouple in the limit $m_{G} \rightarrow 0$, giving rise to a real graviton-mass discontinuity. This phenomenon was discovered by van Dam, Veltman [13] and Zakharov [14] (vDVZ), by analyzing the one-graviton exchange amplitude. In particular, they found that if the graviton has a small mass, no matter how small, a finite difference in the deflection angle for the light-bending from the sun would be predicted with respect to the massless case. Then, since this prediction is out of $25 \%$ from the measured value (which is in agreement with the general relativity predictions), they concluded that the massive gravity theory can be ruled out by solar system observations. This conclusion relies on the fact that the terms singular in $m_{G}$ vanish in the one-graviton approximation. However, there are criticisms on the validity of this approximation based on the observation that higher order corrections can be singular in the graviton mass and therefore cannot be neglected [15, 16].

Here we show that there exists another aspect of the vDVZ discontinuity, to our knowledge not considered so far, concerning the flavor-changing contribution to the Newton potential in the massive gravity theory. In order to see that, let us consider the amplitude in Eq.(16) in the case of one massive-graviton exchange. It can be easily obtained from Eq.(19) with $C=2 / 3$ giving

$$
\begin{gathered}
\mathcal{M}=\frac{G_{F}}{16 \pi^{2} \sqrt{2} \bar{M}_{P}^{2}} \sum_{f} \lambda_{f} \bar{u}\left(p_{2}\right)\left\{\frac{q^{2}}{\left(q^{2}-m_{G}^{2}-i \varepsilon\right)}\left(\left(\gamma^{\mu} p^{\nu}+\gamma^{\nu} p^{\mu}\right) P_{L}-\frac{2}{3} \eta^{\mu \nu} M_{+}\right) g_{a}\left(x_{f}\right)\right. \\
\left.+\frac{2}{3} \eta^{\mu \nu} M_{-} \frac{(p \cdot q)}{\left(q^{2}-m_{G}^{2}-i \varepsilon\right)} g_{a}\left(x_{f}\right)\right\} u\left(p_{1}\right) \hat{T}_{\mu \nu}^{\mathrm{ext}} .
\end{gathered}
$$

A remarkable aspect of Eq.(21) is that the $1 / q^{2}$ pole in the last term does not vanish in the limit $m_{G} \rightarrow 0$. As a consequence it generates an off-diagonal contribution (in flavor space) to the Newton potential. In order to see that, one has to first look at the nonrelativistic limit of Eq.(21) in the case of an external heavy gravitational source. This should be compared with the Born approximation to the corresponding scattering amplitude in nonrelativistic quantum mechanics written in terms of the potential function $V(r)$. Then, the matrix element of the Newton potential $V(r)$ (generated by a heavy massive particle $M$ ) between initial and final fermions states is

$$
\langle j|V(r)| i\rangle=-\frac{G_{N} M}{r} e^{-r m_{G}}\left(m_{i} \delta_{i j}+\left(m_{i}-m_{j}\right) \Delta_{i j}\right)
$$

with

$$
\Delta_{i j}=\frac{G_{F}\left(m_{i}^{2}-m_{j}^{2}\right)}{32 \pi^{2} \sqrt{2}} \sum_{f} K_{f i} K_{f j}^{\star} g_{a}\left(x_{f}\right)
$$


where $G_{N}$ is the Newton constant for the massive-graviton case [13] and $\delta_{i j}$ is the delta-function in flavor space, the indices $i$ and $j$ standing for the ingoing and outgoing fermion states respectively. The discontinuity in $m_{G}$ is manifest in the fact that in the limit $m_{G} \rightarrow 0$ the $\Delta_{i j}$ term does not vanish, while the $\Delta_{i j}$ is strictly zero in the case of massless graviton. Notice that the FC contribution to the Newton potential vanishes in the case of equal masses $\left(m_{i}=m_{j}\right)$ and its attractive or repulsive nature is related to the sign of the $\sum_{f} K_{f i} K_{f j}^{\star} g_{a}\left(x_{f}\right)$ quantity. It should be recalled that the result of Eq.(22) has been derived in the one-graviton approximation and therefore all the criticisms with respect to the vDVZ discontinuity apply also to it [15, 16].

\section{Flavor-changing processes with massive spin-2 parti- cles}

In this section we analyze a few applications of the results derived in Secs. 2 and 3 related to processes that are characterized by the kinematical regimes of small and large values of $\left|q^{2}\right| / m_{W}^{2}$. At this aim, we consider a scenario containing an elementary massive spin-2 particle in the spectrum coupled to a conserved energy-momentum tensor $T_{\mu \nu}(x)$ via the interaction Lagrangian

$$
\mathcal{L}_{\text {eff }}=-\frac{1}{\Lambda} T_{\mu \nu}(x) G^{\mu \nu}(x)
$$

where $G_{\mu \nu}(x)$ represents the massive spin-2 field and $\Lambda$ is a mass-scale free parameter having no relation with the Plank mass $\bar{M}_{P}$. For the spin-2 free Lagrangian we take the Pauli-Fierz action which is the ghost-free linearized action for a massive spin-2 particle [13. Since we will consider here only the first order in the $1 / \Lambda$ expansion, self-interactions of the spin- 2 fields can be neglected and the $T_{\mu \nu}(x)$ is reduced to the energy-momentum tensor of matter fields in flat space-time. Then, the off-diagonal one-loop correction to the Lagrangian in Eq.(24) can be easily generalized from the gravitational case by replacing $\bar{M}_{P} \rightarrow \Lambda$.

We consider first the case of a decay process

$$
f_{1}\left(p_{1}\right) \rightarrow f_{2}\left(p_{2}\right) G(q)
$$

that can be considered as the spin-2 counterpart of the well-known fermion radiative decay $f_{1} \rightarrow f_{2} \gamma$. In Eq.(25) $f_{1}$ and $f_{2}$ stand for two generic different fermions of the same charge with $p_{1,2}$ the corresponding momenta, $q=p_{1}-p_{2}$ and all the external particles are assumed to be much lighter than the $\mathrm{W}$ boson and the fermions running into the loop. The corresponding decay amplitude can be written as:

$$
M\left(f_{1} \rightarrow f_{2} G\right)=-\frac{i m_{G}^{2} G_{F}}{16 \pi^{2} \sqrt{2} \Lambda} \sum_{f} \lambda_{f} g_{a}\left(x_{f}\right) \bar{u}_{2}\left(p_{2}\right)\left[\gamma^{\mu} p^{\nu}+\gamma^{\nu} p^{\mu}\right] P_{L} u_{1}\left(p_{1}\right) \epsilon_{\mu \nu}^{i}(q)
$$


where $\epsilon_{\mu \nu}^{i}(q)$ is the polarization tensor of the spin-2 particle of mass $m_{G}$ with the index $i=1,5$ labeling the five independent polarizations and the function $g_{a}(x)$ is defined in Eq.(13). In obtaining Eq.(26) we have used the fact that for an on-shell spin-2 particle one has $q_{\mu} \epsilon^{\mu \nu}(q)=0$ and $\eta_{\mu \nu} \epsilon^{\mu \nu}(q)=0$.

We point out that in the limit $m_{G} \rightarrow 0$ the amplitude in Eq.(26) seems to vanish, being proportional to $m_{G}^{2}$. However, the sum over polarizations of a massive spin-2 particle [13, 11]

$$
\sum_{i=1}^{5} \epsilon_{\mu \nu}^{i}(q) \epsilon_{\alpha \beta}^{i \dagger}(q)=\frac{1}{2}\left(\tilde{\eta}^{\mu \alpha} \tilde{\eta}^{\nu \beta}+\tilde{\eta}^{\nu \alpha} \tilde{\eta}^{\mu \beta}-\frac{2}{3} \tilde{\eta}^{\mu \nu} \tilde{\eta}^{\alpha \beta}\right)
$$

contains terms singular as $m_{G} \rightarrow 0$. Thus, after summing over all the polarizations, averaging over the initial ones, and integrating over the phase space, the decay width, assuming $f_{2}$ massless, reads:

$$
\Gamma\left(f_{1} \rightarrow f_{2} G\right)=\frac{G_{F}^{2} m_{1}^{7} f\left(x_{G}\right)}{192(2 \pi)^{5} \Lambda^{2}}\left|\sum_{f} \lambda_{f} g_{a}\left(x_{f}\right)\right|^{2}
$$

where $x_{G}=m_{G}^{2} / m_{1}^{2}$, and $f(x)=(1-x)\left(1-\frac{3}{2}\left(x+x^{2}+x^{4}\right)+\frac{7}{2} x^{3}\right)$ absorbs the matrix element and phase space corrections.

These results can have an application in the framework of quantum gravity propagating in large extra dimensions [17, 18]. In [17, 18], it was pointed out that if compactified extra dimensions exist, with only gravity propagating in the bulk, the fundamental scale of quantum gravity could be much lower than the Plank scale $M_{P}$. In this scenario, the standard Newton constant $G_{N}$ in $(3+1)$-dimensional space is related to the corresponding Plank scale $M_{D}$ in $(D=4+\delta)$-dimensional space, by

$$
G_{N}^{-1}=8 \pi R^{\delta} M_{D}^{2+\delta}
$$

where $R$ is the radius of the compactified manifold assumed here to be on a torus. If one requires $M_{D} \sim \mathrm{TeV}$, present tests on gravity law imply that $\delta \geq 2$.

After integrating out compact extra dimensions, the effective low energy theory describes an almost continuous spectrum of massive spin-2 particles, which are excitations of the standard graviton field. Then, each massive spin-2 field is coupled to the matter field by Eq.(24), where the energy scale $\Lambda$ corresponds to the reduced Plank mass $\bar{M}_{P}$. In the case of $M_{D} \sim \mathrm{TeV}$ and $\delta<4$, the mass splitting between the Kaluza-Klein (KK) excitations is of the order of $\mathrm{KeV}$, and the KK spectrum can be approximated as a continuous. In this case the number density of modes $(d N)$ between $m_{G}$ and $m_{G}+d m_{G}$ of KK spin-2 masses is [11]

$$
d N=S_{\delta-1} \frac{\bar{M}_{P}^{2}}{M_{D}^{2+\delta}} m_{G}^{\delta-1} d m_{G}
$$


where $S_{\delta-1}$ is the surface of a unit-radius sphere in $\delta$ dimensions which is given by $S_{\delta-1}=$ $2 \pi^{n} /(n-1)$ ! and $S_{\delta-1}=2 \pi^{n} / \prod_{k=0}^{n-1}\left(k+\frac{1}{2}\right)$ ! for $\delta=2 n$ and $\delta=2 n+1$, with $n$ integer, respectively.

In this framework, we consider the inclusive flavor-changing graviton decay $f_{1} \rightarrow f_{2} G$, where $G$ stands for any KK massive spin- 2 gravitons with mass $m_{G}<m_{1}$. In this case, the corresponding decay width is obtained by multiplying Eq.(28) for $d N$, with $\Lambda$ replaced by $\bar{M}_{P}$, and integrating it over all the allowed kinematical range of $m_{G}$. In particular, for the inclusive decay we get

$$
\sum_{G} \Gamma\left(f_{1} \rightarrow f_{2} G\right)=\frac{G_{F}^{2} m_{1}^{7+\delta} S_{\delta-1} I(\delta)}{192(2 \pi)^{5} M_{D}^{2+\delta}}\left|\sum_{f} \lambda_{f} g_{a}\left(x_{f}\right)\right|^{2}
$$

where $I(\delta)=960 /(\delta(2+\delta)(6+\delta)(8+\delta)(10+\delta))$ and $\delta \geq 2$. As we can see from Eq.(31), the integration over the number of KK states cancels the $1 / \bar{M}_{P}^{2}$ suppression factor of the single graviton emission. We stress that the final KK gravitons are detected as missing energy, since for laboratory experiments they can be approximated as stable particles [18. This is due to the fact that the decay width of a single KK graviton is strongly suppressed by $1 / \bar{M}_{P}^{2}$.

For a numerical evaluation of Eq. (31) we restrict ourselves to the inclusive B-meson decay $B_{d} \rightarrow X_{s} G$, where the standard GIM suppression is enhanced due to the contribution of the top-quark running in the loop. In particular, for $\delta=2$, the corresponding branching-ratio, normalized to the experimental $B R(B \rightarrow X \bar{\nu} e) \simeq 10.4 \%$ of the semileptonic decay, is

$$
\sum_{G} B R\left(B_{d} \rightarrow X_{s} G\right) \simeq 10^{-13}\left(\frac{\mathrm{TeV}}{M_{D}}\right)^{4}\left(\frac{m_{b}}{4.3 \mathrm{GeV}}\right)^{4}
$$

where $X_{s}$ stands for any hadronic state containing an $s$-quark. The above result corresponds to a top-quark mass $m_{t}=171.2 \mathrm{GeV}$.

Next we consider the decay of a massive spin-2 particle $G \rightarrow f_{2} \bar{f}_{1}$, where $f_{1,2}$ are two fermions of different flavor. In the approximation of neglecting the final fermion masses the corresponding amplitude can be obtained from Eq.(14) by setting in it $(p \cdot q)=M_{+}=M_{-}=0$ and replacing the function $g_{a}(x)$ with the function $G_{a}(x, y)$ provided in Appendix B, where the full $q^{2}$ dependence in the form factors is retained, or

$$
M\left(G \rightarrow f_{2} \bar{f}_{1}\right)=-\frac{i G_{F} m_{G}^{2}}{16 \pi^{2} \sqrt{2} \Lambda} \sum_{f} \lambda_{f} G_{a}\left(x_{f}, x_{G}\right) \bar{u}_{2}\left(p_{2}\right)\left[\gamma^{\mu} p^{\nu}+\gamma^{\nu} p^{\mu}\right] P_{L} v_{1}\left(p_{1}\right) \epsilon_{\mu \nu}^{i}(q)
$$

In Eq.(33) $x_{G}=m_{G}^{2} / m_{W}^{2}, p=p_{2}-p_{1}$ and the $v_{1}\left(p_{1}\right)$ is the spinor of the antifermion associated with $\bar{f}_{1}$. Then, for the corresponding decay width we obtain

$$
\Gamma\left(G \rightarrow f_{2} \bar{f}_{1}\right)=\Gamma_{f} \frac{G_{F}^{2} m_{G}^{4}}{64 \pi^{4}}\left|\sum_{f} \lambda_{f} G_{a}\left(x_{f}, x_{G}\right)\right|^{2}
$$


where

$$
\Gamma_{f}=\frac{m_{G}^{3} N_{c}}{160 \pi \Lambda^{2}}
$$

is the tree-level decay width of a massive spin-2 particle into a pair of massless fermions of the same flavor, with $N_{c}$ the color factor.

Because of the presence of the $G_{F}^{2} m_{G}^{4}$ term in the numerator of Eq.(34), one would naïvely expect that when $m_{G} \gg m_{W}$, the one-loop decay would be strongly enhanced. However, this is not the case. Indeed, by using the asymptotic expansion of the $\mathrm{B}_{0}(y)$ and $\mathrm{C}_{0}(x, y)$ functions at large values of $y$ [19], we obtain

$$
\begin{aligned}
G_{a}(x, y) & =\frac{1}{y}\left[F(x)-\frac{x}{2}(\log (y)-i \pi)\right]+\bar{G}(y)+\mathcal{O}\left(\frac{1}{y^{2}}\right) \\
F(x) & =\left(\frac{3 x(3-x)}{4(1-x)}+\frac{x\left(8-6 x+x^{2}\right) \log (x)}{2(x-1)^{2}}\right)-4\left(\operatorname{Li}_{2}(1-x)-\frac{\pi^{2}}{6}\right)
\end{aligned}
$$

where $\operatorname{Li}(\mathrm{x})$ stands for the usual dilogarithm function and $\overline{\mathrm{G}}(y)$ is a pure function of $y$ whose leading term is proportional to $\log ^{2}(y) / y$, showing that no powerlike enhancement is present in the FC decay when $m_{G} \gg m_{W}$. Furthermore, due to the unitarity of CKM, the contribution of $\overline{\mathrm{G}}(y)$ vanishes when the sum over all internal fermion masses is performed, so that in the limit $m_{G} \gg m_{W}$ the decay width reads:

$$
\left.\Gamma\left(G \rightarrow f_{2} \bar{f}_{1}\right)\right|_{m_{G} \gg m_{W}}=\Gamma_{f} \frac{G_{F}^{2} m_{W}^{4}}{64 \pi^{4}}\left|\sum_{f} \lambda_{f}\left(F\left(x_{f}\right)-\frac{x_{f}}{2}\left(\log \left(\frac{m_{G}^{2}}{m_{W}^{2}}\right)-i \pi\right)\right)\right|^{2} .
$$

As a last example, we consider the high-energy limit of the following FC fermion scattering process

$$
f_{i}\left(p_{1}\right) X\left(q_{1}\right) \rightarrow f_{j}\left(p_{2}\right) X\left(q_{2}\right)
$$

induced by the one-graviton exchange amplitude, where $f_{i, j}$ stands for two fermions of different flavor and $X$ indicates a generic particle. Because of the fact that gravity does not change flavor at tree level, only the t-channel diagram will contribute to the above scattering (where $\left.t \equiv\left(p_{1}-p_{2}\right)^{2}\right)$, provided the $X$ particle is different from the initial and final fermions. In the fermion massless limit, only the first term in Eq.(19), proportional to the $G_{a}(x, y)$ function, will contribute to the matrix element and therefore, regardless the graviton is assumed massive or massless, in the region of large $t$ values $\left(|t| \gg m_{W}^{2}\right)$, the following relation among cross sections holds:

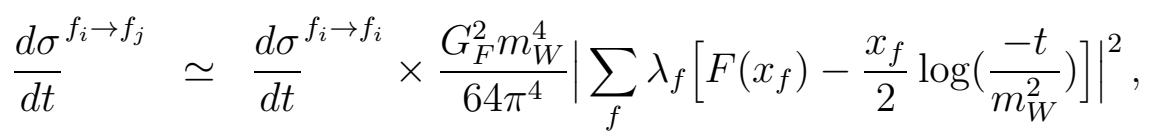

where $\frac{d \sigma}{d t}^{f_{i} \rightarrow f_{i}}$ stands for the diagonal part (in flavor) of the tree-level differential cross section of the process $f_{i} X \rightarrow f_{i} X$ mediated by the one-graviton exchange diagram. 


\section{Conclusions}

We computed the one-loop flavor-changing quark-graviton vertex verifying all the WI induced by the conservation of energy-momentum tensor. The calculation was performed in the $R_{\xi}$ gauge with $\xi=1$, using a modified version of the 't-Hooft gauge-fixing function, and cross-checked in the unitary gauge. We found that the corresponding form factors turn out to be strongly GIM suppressed when the internal fermion masses are much smaller than the $\mathrm{W}$ mass. These results can be easily generalized to the corresponding FC lepton-graviton vertices, provided the neutrinos acquire mass of the Dirac type.

We investigated the case of a flavor-changing fermion-graviton vertex coupled to an external gravitational source. We show that, due to the angular-momentum conservation, gravity and weak interactions induce at one-loop an effective local interaction for the $\Delta F=1$ transitions. At low energy, the corresponding effective Hamiltonian is given by local operators of dimension eight suppressed by a characteristic scale of order $\Lambda=\sqrt{\bar{M}_{P} m_{W}} \sim 10^{10} \mathrm{GeV}$. Thus gravitational FC effects are completely negligible when compared to the known contribution of $\Delta F=1$ dimension six operators of the SM.

We showed that, in the case of massless graviton, the locality of the FC gravitational interaction is related to the cancellation of $1 / q^{2}$ pole in the corresponding one-graviton exchange amplitude. The latter does not take place if the graviton has a small mass signaling another aspect of the known vDVZ discontinuity in the graviton mass. Indeed we showed that in the graviton-massive case the Newton potential acquires a FC contribution that vanishes in the limit of equal external masses.

As a few applications of our results we analyzed a new physics scenario containing massive spin-2 particles coupled to the SM fields. In this framework, we calculated the width of the FC decay $f_{1} \rightarrow f_{2} G$ and the decay of a heavy spin-2 particle in two fermions of different flavor, $G \rightarrow f_{2} \bar{f}_{1}$. We also considered the scenario of quantum gravity propagating in large extra dimensions. In this framework, we evaluated the inclusive decay width for the process $f_{1} \rightarrow f_{2} G$, where $G$ stands for any Kaluza-Klein spin-2 graviton, and estimated the branching ratio for the inclusive $B_{d}$-meson decay $B_{d} \rightarrow X_{s} G$. Finally, as a consistency test of our results, we studied the asymptotic behavior for large spin-2 masses of the decay $G \rightarrow f_{2} \bar{f}_{1}$ and the high-energy limit of the gravitational scattering $f_{1} X \rightarrow f_{2} X$ (with $X \neq f_{1,2}$ ) with respect to the GIM mechanism. We explicitly check that also in the asymptotic limit, the GIM mechanism acts in the usual way strongly suppressing the process for small internal fermion masses.

The results presented for the massive graviton can be easily generalized to the case of a 
graviscalar, $\phi_{S}$, whose interaction with the SM fields can be described by

$$
\mathcal{L}_{\mathrm{int}}=-\frac{1}{\Lambda_{S}} T_{\mu \nu}(x) \phi_{S}(x) \eta^{\mu \nu}
$$

In particular, the counterpart of Eq.(22) for the graviscalar reads:

$$
\langle j|V(r)| i\rangle=-\frac{1}{\Lambda_{S}^{2}} \frac{M}{4 \pi r} e^{-r m_{\phi_{S}}}\left(m_{i} \delta_{i j}+\left(m_{i}-m_{j}\right) \Delta_{i j}^{S}\right)
$$

with $\Delta_{i j}^{S}=6 \Delta_{i j}$. The width of the FC decay $f_{1} \rightarrow f_{2} \phi_{S}$ neglecting the mass of the produced particles is instead:

$$
\Gamma\left(f_{1} \rightarrow f_{2} \phi_{S}\right)=\frac{G_{F}^{2} m_{1}^{7}}{32(2 \pi)^{5} \Lambda_{S}^{2}}\left|\sum_{f} \lambda_{f} g_{a}\left(x_{f}\right)\right|^{2} .
$$

Finally, because the coupling between a graviscalar and the fermions is proportional to the fermion mass, the decay width of a graviscalar into two massless fermions is null.

\section{Acknowledgments}

We thank G. Dvali, G. Giudice, R. Rattazzi and G. Veneziano for useful discussions. This work was supported in part by an EU Marie-Curie Research Training Network under Contract No. MRTN-CT-2006-035505 (HEPTOOLS) and by the INTAS Project No. 05-1000008-8328.

\section{Appendix A}

In this appendix we report the Feynman rules for gravitational interactions with SM fields that are relevant for the processes considered in this article. They are presented in Fig, 2 . The symbols used in Fig, 2 are defined as:

$$
\begin{aligned}
X_{\mu \nu}^{(f)}= & \gamma_{\mu}\left(k_{1 \nu}+k_{2 \nu}\right)-\eta_{\mu \nu}\left(\not k_{1}+\not k_{2}-2 m_{i}\right) \\
X_{\mu \nu \alpha \beta}^{(V)}= & \frac{1}{2} \eta_{\mu \nu} k_{2 \alpha} k_{1 \beta}+\eta_{\alpha \beta} k_{1 \mu} k_{2 \nu}-\eta_{\mu \beta} k_{1 \nu} k_{2 \alpha}-\eta_{\mu \alpha} k_{2 \nu} k_{1 \beta} \\
& +\left(k_{1} \cdot k_{2}+m_{W}^{2}\right)\left(\eta_{\mu \alpha} \eta_{\nu \beta}-\frac{1}{2} \eta_{\mu \nu} \eta_{\alpha \beta}\right) \\
& +\frac{1}{\xi}\left[\frac{1}{2} \eta_{\mu \nu}\left(k_{1 \alpha} k_{1 \beta}+k_{2 \alpha} k_{2 \beta}+k_{1 \alpha} k_{2 \beta}\right)-\eta_{\nu \beta} k_{1 \alpha} k_{1 \mu}-\eta_{\nu \alpha} k_{2 \beta} k_{2 \mu}\right] \\
X_{\mu \nu}^{(S)}= & \left(k_{1} \cdot k_{2}+\xi m_{W}^{2}\right) \eta_{\mu \nu}-k_{1 \nu} k_{2 \mu}-k_{1 \mu} k_{2 \nu} \\
X_{\mu \nu \alpha}^{(f, V)=} & K_{i j}\left(2 \gamma_{\alpha} \eta_{\mu \nu}-\gamma_{\mu} \eta_{\nu \alpha}-\gamma_{\nu} \eta_{\mu \alpha}\right) P_{L} \\
X_{\mu \nu}^{(f, S)=} & K_{i j}\left(\frac{m_{j}}{m_{W}} P_{R}-\frac{m_{i}}{m_{W}} P_{L}\right) \eta_{\mu \nu} .
\end{aligned}
$$



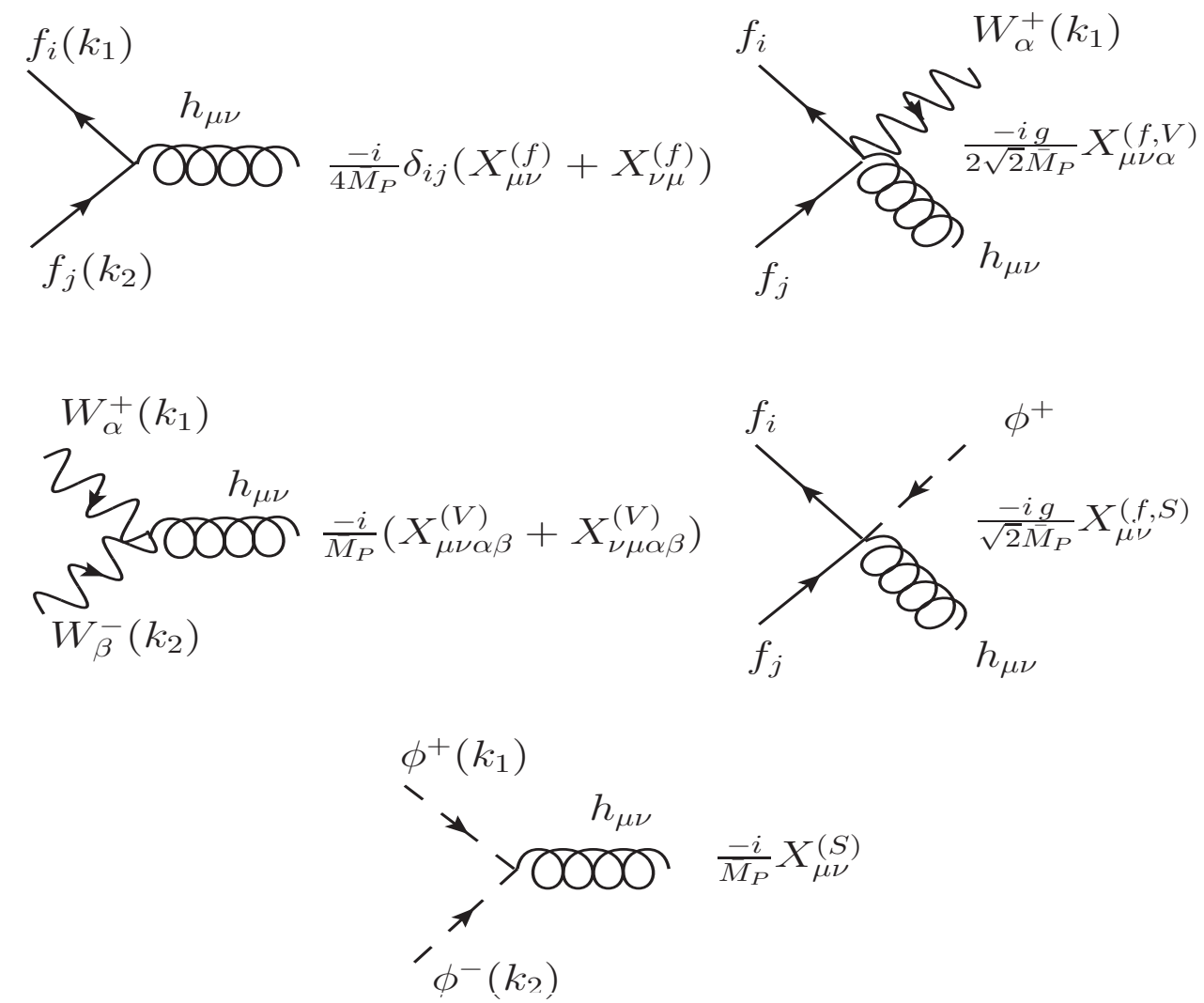

Figure 2: Three and four-point vertex Feynman rules relevant for our calculation. The particle momenta are assumed to flow along the direction of the arrows. For the meaning of the symbols see text.

\section{Appendix B}

In this appendix we discuss the form taken by $\hat{T}^{\mu \nu}$ in the limit $(p \cdot q)=p^{2}+q^{2}=0$, i.e. when terms proportional to $m_{1,2}^{2}$ are neglected. In this limit, from the WI on Eq.(11), only five form factors survive and $\hat{T}^{\mu \nu}$ can be cast in the following form

$$
\hat{T}^{\mu \nu}=\frac{i G_{F}}{16 \pi^{2} \sqrt{2}} \sum_{i=1}^{5} F_{i}(p, q) \bar{u}_{2}\left(p_{2}\right) \widehat{O}_{i}^{\mu \nu} u_{1}\left(p_{1}\right)
$$

where

$$
\begin{aligned}
& \widehat{O}_{1}^{\mu \nu}=\left(\gamma^{\mu} p^{\nu}+\gamma^{\nu} p^{\mu}\right) P_{L}-\frac{p^{\mu} q^{\nu}+q^{\mu} p^{\nu}}{q^{2}} M_{-}, \\
& \widehat{O}_{2}^{\mu \nu}=\left(-\eta^{\mu \nu} q^{2}+q^{\mu} q^{\nu}\right) M_{+},
\end{aligned}
$$




$$
\begin{aligned}
& \widehat{O}_{3}^{\mu \nu}=\left(-\eta^{\mu \nu} q^{2}+q^{\mu} q^{\nu}\right) M_{-}, \\
& \widehat{O}_{4}^{\mu \nu}=\left(p^{\mu} p^{\nu}\right) M_{+}, \\
& \widehat{O}_{5}^{\mu \nu}=\left(p^{\mu} p^{\nu}\right) M_{-} .
\end{aligned}
$$

with $F_{1}(p, q)=f_{1}(p, q), F_{2}(p, q)=f_{5}(p, q), F_{3}(p, q)=f_{9}(p, q), F_{4}(p, q)=f_{4}(p, q)$, and $F_{5}(p, q)=$ $f_{8}(p, q)$. In this basis each element $\widehat{O}_{i}^{\mu \nu}$ satisfies the gauge invariance condition $q_{\mu} \widehat{O}_{i}^{\mu \nu}=0$, and so the corresponding form factors $F_{i}(p, q)$ are manifestly independent.

Writing

$$
\begin{aligned}
& F_{1}(p, q)=-q^{2} \sum_{f} \lambda_{f} G_{a}\left(x_{f}, y\right), \\
& F_{2}(p, q)=-\sum_{f} \lambda_{f} G_{b}\left(x_{f}, y\right), \\
& F_{3}(p, q)=0 \\
& F_{4}(p, q)=\sum_{f} \lambda_{f} G_{c}\left(x_{f}, y\right), \\
& F_{5}(p, q)=0
\end{aligned}
$$

we find

$$
\begin{aligned}
G_{a}(x, y) & =\frac{4}{y^{3}}\left[\left(2-3 x+x^{3}\right)\left(B_{0}(y)-B_{0}(\bar{y})\right)+(x-1)^{3}(2+x)\left(C_{0}(x, y)+\frac{1}{x} C_{0}(\bar{x}, \bar{y})\right)\right. \\
& \left.+(x-1)^{2}(2+x) \log (x)\right]+\frac{1}{y^{2}}\left[(1-x)(2+x)-\frac{1}{3}\left(42-23 x+5 x^{2}\right) B_{0}(\bar{y})\right. \\
& +\frac{4}{3}\left(7-4 x+3 x^{2}\right) B_{0}(y)+(x-1)\left(18-7 x+x^{2}\right) C_{0}(x, y) \\
& \left.+\frac{2}{x}(x-1)\left(4-x+3 x^{2}\right) C_{0}(\bar{x}, \bar{y})+\left(14-3 x+4 x^{2}\right) \log (x)\right] \\
& +\frac{1}{y}\left[\frac{(1+x)(2+x)}{4(1-x)}+\frac{1}{6}(x-34) B_{0}(\bar{y})+\frac{1}{3}(8+x) B_{0}(y)-\left(12-6 x+x^{2}\right) C_{0}(x, y)\right. \\
& \left.+2 x C_{0}(\bar{x}, \bar{y})+\frac{\left(34-45 x+18 x^{2}+2 x^{3}\right) \log (x)}{6(x-1)^{2}}\right]-2 C_{0}(x, y) \\
G_{b}(x, y) & =\frac{4}{y^{3}}\left[\left(2-3 x+x^{3}\right)\left(B_{0}(\bar{y})-B_{0}(y)\right)-(x-1)^{3}(2+x)\left(C_{0}(x, y)+\frac{1}{x} C_{0}(\bar{x}, \bar{y})\right)\right. \\
& \left.-(x-1)^{2}(2+x) \log (x)\right]+\frac{2}{y^{2}}\left[(x-1)(2+x)+\frac{4}{3}\left(3-x+x^{2}\right) B_{0}(\bar{y})\right. \\
& -\frac{1}{3}\left(2+x+9 x^{2}\right) B_{0}(y)+\left(6-7 x+2 x^{2}-x^{3}\right) C_{0}(x, y) \\
& \left.-2(x-1)(1+2 x) C_{0}(\bar{x}, \bar{y})-\left(4+2 x+3 x^{2}\right) \log (x)\right]+\frac{1}{y}\left[\frac{3\left(2-5 x+x^{2}\right)}{2(x-1)}\right.
\end{aligned}
$$




$$
\begin{aligned}
& +\frac{1}{3}(2-5 x) B_{0}(\bar{y})+\frac{2}{3}(8-5 x) B_{0}(y)+(4-2 x) C_{0}(x, y) \\
& \left.-\frac{1}{x}\left(4-6 x+6 x^{2}\right) C_{0}(\bar{x}, \bar{y})-\frac{\left(2+3 x-24 x^{2}+10 x^{3}\right) \log (x)}{3(x-1)^{2}}\right]-2 C_{0}(\bar{x}, \bar{y}) \\
G_{c}(x, y) & =\frac{20}{y^{3}}\left[\left(2-3 x+x^{3}\right)\left(B_{0}(\bar{y})-B_{0}(y)\right)-(x-1)^{3}(2+x)\left(C_{0}(x, y)+\frac{1}{x} C_{0}(\bar{x}, \bar{y})\right)\right. \\
& \left.-(x-1)^{2}(2+x) \log (x)\right]+\frac{2}{y^{2}}\left[8(x-1)(2+x)+\frac{1}{3}\left(78-59 x+5 x^{2}\right) B_{0}(\bar{y})\right. \\
& -\frac{1}{3}\left(10-25 x+39 x^{2}\right) B_{0}(y)+18(x-2)(x-1) C_{0}(x, y)-18 x(x-1) C_{0}(\bar{x}, \bar{y}) \\
& \left.-\left(26+3 x+13 x^{2}\right) \log (x)\right]+\frac{1}{y}\left[\frac{3\left(2-5 x+x^{2}\right)}{x-1}+\frac{4(10-x) B_{0}(\bar{y})}{3}\right. \\
& +\frac{4}{3}(2-5 x) B_{0}(y)+2(9-x)(2-x) C_{0}(x, y)-\frac{1}{x}\left(4-14 x+18 x^{2}\right) C_{0}(\bar{x}, \bar{y}) \\
& -\frac{\left.2\left(20-24 x-15 x^{2}+10 x^{3}\right) \log (x)\right]+4 C_{0}(x, y)-2 C_{0}(\bar{x}, \bar{y}),}{3(x-1)^{2}}
\end{aligned}
$$

where $x_{f} \equiv m_{f}^{2} / m_{W}^{2}$ and $y \equiv q^{2} / m_{W}^{2}$, and $\bar{x} \equiv 1 / x, \bar{y} \equiv y / x$, and the functions $B_{0}(y), C_{0}(x, y)$ are defined as

$$
\begin{aligned}
B_{0}(y) & =\frac{2}{y}\left(y-\sqrt{y(4-y)} \arctan \left[\frac{y}{\sqrt{y(4-y)}}\right]\right) \\
C_{0}(x, y) & =\int_{0}^{1} d x_{1} \int_{0}^{1-x_{1}} d x_{2}\left[(1-x)\left(x_{1}+x_{2}\right)+y x_{1} x_{2}-1\right]^{-1} .
\end{aligned}
$$

An analytic result for the function $C_{0}(x, y)$ can be obtained from Refs. [20, 19]. The functions $G_{a, b, c}(x, y)$ generalize the $g_{a, b}(x)$ in Eq.(13) to include the full $q^{2}$ dependence and satisfy the following conditions

$$
\lim _{y \rightarrow 0} G_{a}(x, y)=g_{a}(x), \quad \lim _{y \rightarrow 0} G_{b}(x, y)=g_{b}(x), \quad \lim _{y \rightarrow 0} G_{c}(x, y)=0 .
$$

Notice that, despite the presence of pure $\log (x)$ terms (not multiplied by $x$ ) in Eqs.(49)-(51), the $G_{a . b . c}(x, y)$ functions turn out to be of order $\mathcal{O}(x)$ for small $x$. This is because the $\log (x)$ terms cancel out when summed to the corresponding terms proportional to the $B(\bar{y})$ function. Indeed, $B(\bar{y})=\log (x)+\mathcal{O}(x)$. Therefore, as expected by the infrared behavior of the diagrams in Fig. 1, for small internal fermion masses, the GIM mechanism strongly suppresses the TFCNC in all ranges of $q^{2}$. 


\section{References}

[1] R. Delbourgo and P. Phocas-Cosmetatos, Lett. Nuovo Cim. 5, 420 (1972); Phys. Lett. B 41, 533 (1972).

[2] M. T. Grisaru, P. van Nieuwenhuizen and C. C. Wu, Phys. Rev. D 12, 1813 (1975).

[3] F.A. Berends and R. Gastmans, in Proceedings of the Fifth International Conference on Neutrino Science, edited by A. Frenkel and G. Marx (Central Research Institute for Physics, Budapest, Hungary, 1975), p.310.

[4] I. Y. Kobzarev and L. B. Okun, Zh. Eksp. Teor. Fiz. 43 (1962) 1904 [Sov. Phys. JETP 16 (1963) 1343].

[5] K. A. Milton, Phys. Rev. D 15 (1977) 2149; Phys. Rev. D 15, 538 (1977).

[6] F. A. Berends and R. Gastmans, Annals Phys. 98, 225 (1976).

[7] C. M. DeWitt and B. S. DeWitt, Physics 1, 3 (1964).

[8] R. Contino, L. Pilo, R. Rattazzi and A. Strumia, JHEP 0106, 005 (2001) arXiv:hep-ph/0103104].

[9] T. Han, J. D. Lykken and R. J. Zhang, Phys. Rev. D 59, 105006 (1999) arXiv:hep-ph/9811350.

[10] J. F. Nieves and P. B. Pal, Phys. Rev. D 72 (2005) 093006 arXiv:hep-ph/0509321.

[11] G. F. Giudice, R. Rattazzi and J. D. Wells, Nucl. Phys. B 544, 3 (1999) arXiv:hep-ph/9811291.

[12] D. Binosi and L. Theussl, Comput. Phys. Commun. 161 (2004) 76 arXiv:hep-ph/0309015.

[13] H. van Dam and M. J. G. Veltman, Nucl. Phys. B 22 (1970) 397.

[14] V. I. Zakharov, JETP Lett. 12 (1970) 312 [Pisma Zh. Eksp. Teor. Fiz. 12 (1970) 447].

[15] A. I. Vainshtein, Phys. Lett. B 39, 393 (1972).

[16] C. Deffayet, G. R. Dvali, G. Gabadadze and A. I. Vainshtein, Phys. Rev. D 65, 044026 (2002) arXiv:hep-th/0106001; G. Dvali, arXiv:hep-th/0402130.

[17] N. Arkani-Hamed, S. Dimopoulos and G. R. Dvali, Phys. Lett. B 429, 263 (1998) arXiv:hep-ph/9803315. 
[18] I. Antoniadis, N. Arkani-Hamed, S. Dimopoulos and G. R. Dvali, Phys. Lett. B 436, 257 (1998) arXiv:hep-ph/9804398.

[19] M. Roth and A. Denner, Nucl. Phys. B 479, 495 (1996) arXiv:hep-ph/9605420.

[20] G. 't Hooft and M. J. G. Veltman, Nucl. Phys. B 153, 365 (1979); G. J. van Oldenborgh and J. A. M. Vermaseren, Z. Phys. C 46, 425 (1990). 